\title{
Mechanisms of reproductive aging in the females
}

\author{
DENG ManQi \\ Brigham and Women's Hospital, Harvard Medical School, Boston, MA 02115, USA
}

Received April 22, 2012; accepted June 28, 2012

\begin{abstract}
Aging is a developmental process occurring in all living organisms after reaching a critical developmental stage, characterized by progressive loss of functions until death. Different cells/tissues age differently depending on epigenetics and cell-cell interactions. While males maintain fertility for the most part of their life females only maintain reproductive ability for a short time compared with their lifespan. The interesting question is why and how the females lose fertility so quickly. There have been many hypotheses proposed from different perspectives and recent research has revealed unusual interactions between germ cells and somatic cells which may determine the lifespan of reproduction in the females. This review briefly discusses recent progress in reproductive aging in the well studied model, C. elegans, and focuses on the molecular mechanisms which may be conserved across all animals including humans.
\end{abstract}

aging, females, oocytes, lifespan, growth factors, mTOR

Citation: Deng M Q. Mechanisms of reproductive aging in the females. Sci China Life Sci, 2012, 55: 653-658, doi: 10.1007/s11427-012-4351-6

All living organisms undergo programmed aging according to their genetically timed clock and environmental factors. The most amazing aspect of aging is reproductive aging in the females, as manifested by a decline and cessation of reproduction long time before somatic aging. Different from males, females start to show a significant decline of reproduction after reaching to $1 / 3$ of their lifespan, long before aging in the soma. The common features of reproductive aging in different animals include progressive loss of oocyte number and quality, frequent chromosome segregation errors which leads to aneuploidy, dysfunction of mitochondrial and loss of endocrinological function of the ovaries $[1,2]$.

\section{A fast ticking clock for a female's reproduc- tive life}

It is generally accepted that the total number of the oocytes in a mammalian female is determined by finite mitotic divi-

email: mdeng4@partners.org sions of the primordial germ cells during embryo development prior to the start of meiosis and after that the ovaries fail to generate any new oocytes under the physiological conditions $[3,4]$. Therefore, the total number of oocytes in a given female is established before birth. The oocytes and the surrounding somatic cells form functional complexes, e.g., follicles, which either undergo maturation and develop to ovulation or programmed death via atresia. During the aging process, both the number and the quality of the oocytes in the ovaries decline and reach to a point beyond that no more viable offspring can be produced and the associated cyclic endocrinological activities cease, entering the so-called menopause in old women. Therefore, there are two pathways to the exhaustion of the follicle reserve: (i) cyclic ovulation and (ii) programmed oocyte death [5]. The vast majority of the oocytes are destined to death and only a small portion of the oocytes make it to ovulation. In humans, there are over million primordial follicles but only 400 oocytes are dedicated for ovulation throughout the life time $[6,7]$. The mechanism of oocyte death is poorly understood and proposed to play an important role in re-allocating the 
limited resources to ensure the survival and quality of the luckily survived oocytes [8]. It is unclear whether artificial intervention of the programmed apoptosis to "rescue" the follicles from apoptosis could slow down the rate of oocyte depletion in the ovaries and therefore prolongs the reproductive lifespan in the females. Work from $C$. elegans shows that genetic mutations that block all cell deaths during aging disrupt asymmetric resource re-location among the oocytes and compromise the oocyte quality [8], suggesting that apoptosis may be required for producing healthy oocytes which are competent for fertilization and post-fertilization development.

Members of the B cell lymphoma/leukemia (BCL) protein family, including BCL-2 and BAX, have been suggested to be involved in germ cell apoptosis [9-11]. It has been shown in mice that knockout of BAX increases the number of germ cells in fatal ovaries [12,13]. In addition, NANOS3 and DND1 can protect primordial germ cells from apoptosis $[14,15]$. Interestingly, overexpression of BCL-2 reduces follicular apoptosis in the mice [16]. Consistent with the in vivo studies, a prominent decrease of BCL-2 is observed in eggs aged in vitro [17]. More impressively, the ovarian function and reproductive lifespan in the mice are prolonged after disruption of BAX [18]. Consistent with this, damaged oocytes in mice exhibit elevated expression of BAX [19]. It is unclear and would be interesting to see whether $\mathrm{BAX}$ can be a therapeutic target for oocyte rejuvenation in old women.

In mammalian animals, including humans, the reserve of primordial follicle pool determines the length of reproductive age $[3,20,21]$. The activation and inhibition of the primordial follicles determine the rate of follicle depletion in the ovaries [22]. Recent work shows that 3-phosphoinositidedependent protein kinase-1 (PDK1) is involved in maintaining the ovarian follicle pool $[22,23]$. Both PDK1 and PTEN are important regulators of the phosphatidylinositol 3-kinase (PI3K) signaling pathway [24] and inhibition of this pathway by knockout of either PTEN or PDK1 results in over activation and quick depletion of the primordial follicle pool in early adulthood [23,25]. Taken together, many inhibitory factors in the ovaries, such as PDK1 and PTEN, prevent follicle activation and thus contribute to maintaining the follicle pool and antagonizing these inhibitory factors accelerates the exhaustion of the finite follicle reserve [22].

It is worthy of noting that a few laboratories have reported isolation of germ stem cells from adult ovaries, which were shown to support de novo oogenesis in adult ovaries [26,27]. However, these reports are very controversial [28-32] and require independent verification by more laboratories. More careful work needs to be done to formally revise the well-held view that the mammals are born with a finite number of eggs that declines with ageing. It may be possible that postnatal mammalian ovaries may preserve the stemness of a few germ cells, which are usually dormant under physiological conditions and can be activated for neo-oogenesis under experimental conditions. However, more advanced cell tracking techniques are needed to verify that the newly formed oocytes are indeed from the externally introduced germ stem cells not from the residual primordial follicles. While completing this review, a new report using more advanced cell lineage tracking technology shows that mouse postnatal ovaries do not support neo-oogenesis [33], upholding the traditional view. At this moment, it is still too early to say whether aged adult ovaries can be rejuvenated by either awakening the "dormant germ stem cells" or introducing new germ stem cells into old ovaries.

\section{A trade-off between reproduction and life- span?}

There has been a popular theory which proposes that reproduction relocates the limited resources for cell survival and shortens lifespan of the animals [34-37]. From an evolutionary point of view, investment in reproduction, a new conception may risk one's own survival, future reproductive opportunities, and/or current offspring survival. Evidence from many lower species seems to support the trade-off theory. The earlier the reproduction, the shorter the lifespan. Postponing or elimination of reproduction extends the lifespan of the animals [34]. Some studies have reported a positive correlation between maternal age at reproduction and female longevity [35,38-40]. In C. elegans, laser ablation of germ cells in the gonad significantly extends lifespan [41]. However, when the entire somatic gonad is removed, the worm has a normal lifespan. Thus, sterility alone does not lengthen life and the signals that extend lifespan come from somatic gonad. However, reproduction and somatic lifespan can be uncoupled. In humans, reproduction does not seem to significantly shorten women's lifespan $[42,43]$. An increasing trend is that women live longer and longer after cessation of reproduction and entry into menopause. The biological explanation for this phenomenon is that women with a prolonged post-reproductive lifespan have more grandchildren, and hence greater fitness for the population [40]. The benefits of prolonged post-reproductive lifespan may be for the grandmother to take care of their grand children, and thus has evolution advantages, the so-called "grandmother effect" [40,44].

Work from $C$. elegans shows further that the effect of longevity by ablation of the germ cells is through triggering the nuclear localization and activation of the DAF-16/ FOXO transcription factor in the intestine [41]. Interestingly, the reproductive system communicates with the intestine through lipophilic-hormone signaling and that a gene called kri-1 is likely to act in the intestine to promote DAF-16 nuclear localization in response to this signal [45]. It is proposed that $C$. elegans's lifespan is influenced by counter- 
balancing cues from the reproductive system: signals from proliferating germ cells reduce longevity, while signals from the somatic gonad promote a longer life (Figure 1) $[41,46]$. However, significant differences between $C$. elegans and mammals should be noted as the female worms have germ stem cells which undergo continuous self-renewal and differentiation while mammals usually do not. In addition, mammalian animals have developed a more complex hypothalamus-pituitary-gonad system to regulate reproduction (and immune system). Due to the complexity of the reproductive system and regulation in mammalian animals, whether and how reproduction affects the longevity of a female requires further studies.

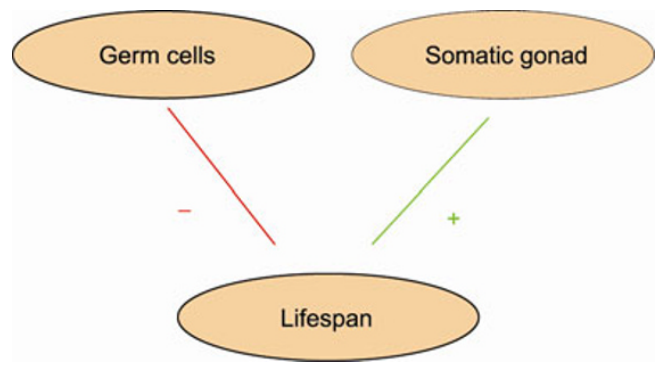

Figure 1 Regulation of longevity by germ cells and somatic gonad. The signals from the germ cells (shown in red line) negatively affect lifespan and the signals from the somatic gonad (shown in green) extend lifespan.

\section{Genetic network underlying the reproductive longevity}

It was highly debated whether aging is genetically controlled or a stochastic event. However, it now seems clear that aging is a developmental event and is genetically regulated. Activation and suppression of some genes have significant influences on longevity. The most striking evidence came from $C$. elegans showing that suppression of insulin/insulin-like growth factor (IGF) pathway significantly extends the worm's lifespan as well as reproductive span $[47,48]$. Recent reports further demonstrate that both insulin/IGF and transforming growth factor (TGF)- $\beta$ signals regulate the speed of reproductive aging [49]. It has been shown that loss of function mutations in either insulin/IGF or TGF- $\beta / \mathrm{Sma} / \mathrm{Mab}$ signal transduction pathways extends reproductive lifespan by improving the oocyte quality in older females [49]. Notably, Sma/Mab regulation of reproductive span is genetically independent of Insulin/IGF signaling (IIS) and dietary restriction [50]. TGF- $\beta$ regulates reproductive aging separately from the developmental regulation of growth, both temporally and transcriptionally [49]. Wild-type $C$. elegans reproduction declines with age, but reduced insulin/IGF signaling (IIS) delays reproductive cessation $[51,52]$. Interestingly, reduced TGF- $\beta / \mathrm{Sma} / \mathrm{Mab}$ signaling extends reproductive lifespan independent of insulin/IGF signaling, caloric restriction, sperm contribution, and ovulation rate [50]. Together, these data suggest that oocyte quality, as defined by chromosome segregation fidelity, fertilizability, and morphology, declines with age in $C$. elegans, and that reduced TGF- $\beta$ Sma/Mab and IIS signaling delay this decline [49]. It has been shown that the TGF- $\beta$ pathway regulates reproductive aging through an interaction between the soma and germ cells [49]. The signals from the germ cells and the somatic gonad regulate longevity $[41,53,54]$, suggesting a bi-directional crosstalk between somatic and reproductive tissues in regulation of aging. The current research seems to suggest two paralleled pathways from neuron-produced ligands to reproductive aging: (i) insulin/IGF-like ligands; (ii) TGF/Sma/Mab ligands. The former target subcutaneous tissues and the later target intestine and muscle to indirectly regulate somatic gonad and germ cells and longevity (Figure 2). Of noted, several TGF- $\beta$ signaling genes are upregulated with age in mouse oocytes [55], paralleling to the observations on the extension of reproductive lifespan in $C$. elegans TGF- $\beta$ mutants [49]. The condensin SMC declines in both mouse and human oocytes with advancing maternal age [55,56], suggesting that chromosome segregation is a key determinant of oocyte quality.

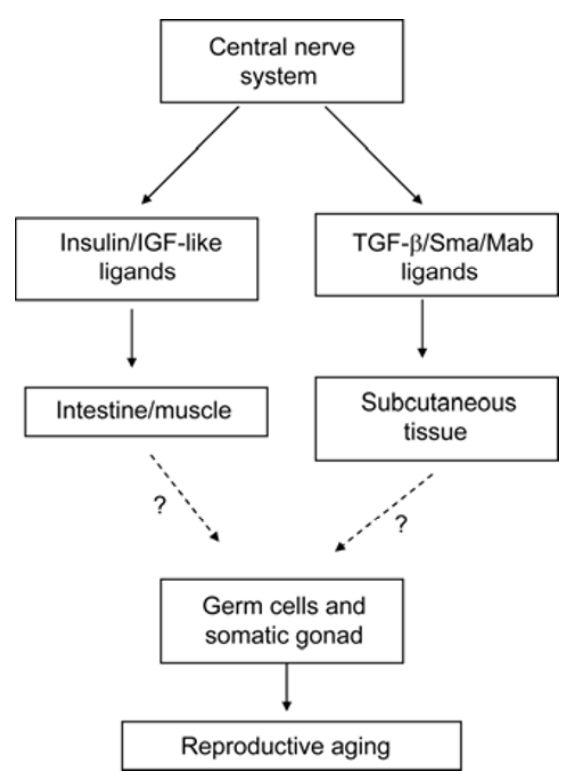

Figure 2 Schematic view of the signaling pathways to reproductive aging. Solid arrows represent the currently identified pathways and the dotted arrows indicate the unknown secondary signals from intestine, muscle and subcutaneous tissues to the germ cells and somatic gonad.

The key unanswered questions, however, are what are the secondary signals generated by the initial insulin/IGF and TGF/Sma/Mab signaling that directly regulate reproductive aging? To what extent the knowledge discovered in worms, flies and even mice are applicable to humans? It is well known that reducing insulin/IGF signaling in mammals leads to diabetic disease and not longevity. Knockout of 
TGF- $\beta$ receptor results in embryonic lethality and conditional knockout of TGF- $\beta$ I receptor and Smad1-Smd-5 in the ovaries leads to oviduct anatomical defects and granulosa cell tumors $[57,58]$ and no benefits on reproductive aging have been observed. All these suggest that insulin/IGF and TGF- $\beta$ signaling in mammals are more complex than in $C$. elegans and more work needs to be done to determine their function in the regulation of reproductive aging in the mammalian animal models.

\section{4 mTOR signaling and energy metabolism}

The mammalian target of rapamycin (mTOR) is a kinase that responds to many intracellular and extracellular signals, ranging from nutrients and energy status, cellular stress, oxygen levels and growth factors [59,60]. The mTOR signaling pathway integrates both intracellular and extracellular signals and serves as a central regulator of cell metabolism, growth, proliferation and survival $[60,61]$. The finely tuned response of mTOR to these stimuli results in alterations to cell metabolism and cell growth [60] and rapamycin as a mTOR inhibitor has been used in cancel treatment $[62,63]$. The mTOR protein is a $289-\mathrm{kD}$ serine-threonine kinase that belongs to the phospho-inositide 3-kinase (PI3K)-related kinase family and is conserved throughout evolution. mTOR nucleates at least two distinct multi-protein complexes, mTOR complex 1 (mTORC1) and mTOR complex 2 (mTORC2) [64]. mTOR1 is a master regulator of cell growth and metabolism and many pathways can converge to mTOR1 signaling, such as growth factors, which stimulate mTORC1 through the activation of the canonical insulin and Ras signaling pathways, energy status, oxygen levels, amino acids and other cellular conditions and signals [61,65]. Numerous studies have shown that reducing the mTOR signaling and energy metabolism can extend lifespan in many species [66,67]. Studies in mice also show that inhibition of the mTOR signaling pathway by rapamycin increases lifespan in genetically heterogeneous mice [68]. A recent report shows that restriction of calorie intake in mouse diet slows down the aging process in reproduction and improves oocyte quality by preventing aneuploidy and spindle defects [69]. The signaling pathways from mTOR to aging are complex and a simplified network is summarized in Figure 3. It is important to understand the crosstalk and dynamic interactions between insulin/IGF/TGF- $\beta$ signaling and the mTOR pathway and how they coordinate to regulate growth, development and aging in reproduction.

In summary, life and aging are two inseparable events for all living organisms and the process of aging may start soon after the life is generated. Different cells/tissues function and age differently, and the germ cells interact (communicate) with the somatic cells at both the tissue/organ and the systems levels. Although germ cells/oocytes are not absolutely

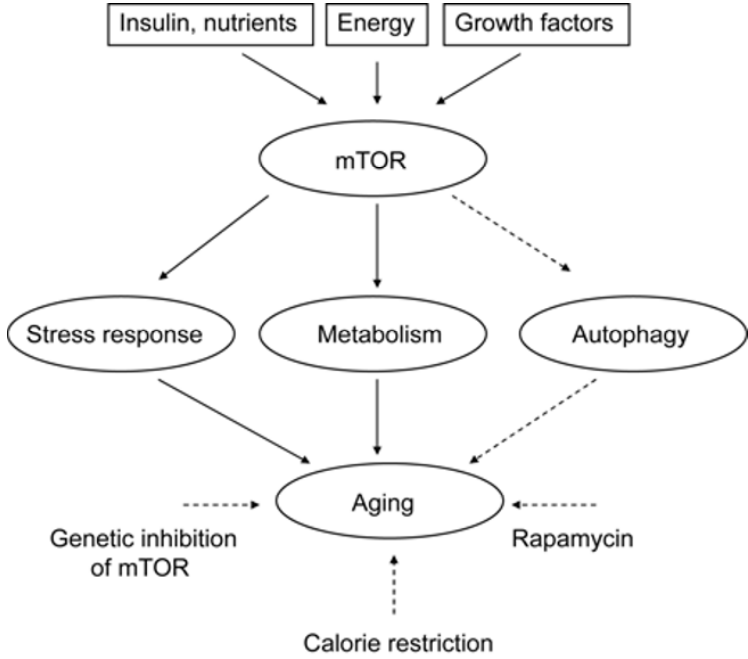

Figure 3 Schematic view of mTOR signaling pathways to aging. The solid arrows indicate the promoting factors for aging. The dotted arrows indicate the inhibition of aging process.

required for the living individual, reproduction is critically important for the survival of the population of the living species. Research in simple organisms has gained important insights into the molecular mechanisms controlling the reproductive aging in the females. It is anticipated that future research will resolve the mysteries of reproductive aging in women, which may provide new treatments to female infertility and to increase the quality of human life.

1 Djahanbakhch O, Ezzati M, Zosmer A. Reproductive ageing in women. J Pathol, 2007, 211: 219-231

2 Hunt P A, Hassold T J. Human female meiosis: what makes a good egg go bad? Trends Genet, 2008, 24: 86-93

3 Gleicher N, Weghofer A, Barad D H. Defining ovarian reserve to better understand ovarian aging. Reprod Biol Endocrinol, 2011, 9: 23

4 Sills E S, Alper M M, Walsh A P. Ovarian reserve screening in infertility: practical applications and theoretical directions for research. Eur J Obstet Gynecol Reprod Biol, 2009, 146: 30-36

5 Gumienny T L, Lambie E, Hartwieg E, et al. Genetic control of programmed cell death in the Caenorhabditis elegans hermaphrodite germline. Development, 1999, 126: 1011-1022

6 Block E. Quantitative morphological investigations of the follicular system in women; variations at different ages. Acta Anat (Basel), 1952, 14: 108-123

7 Baker T G. A quantitative and cytological study of germ cells in human ovaries. Proc R Soc Lond B Biol Sci, 1963, 158: 417-433

8 Andux S, Ellis R E. Apoptosis maintains oocyte quality in aging Caenorhabditis elegans females. PLoS Genet, 2008, 4: e1000295

9 Felici M D, Carlo A D, Pesce M, et al. Bcl-2 and Bax regulation of apoptosis in germ cells during prenatal oogenesis in the mouse embryo. Cell Death Differ, 1999, 6: 908-915

10 Willis S, Day C L, Hinds M G, et al. The Bcl-2-regulated apoptotic pathway. J Cell Sci, 2003, 116: 4053-4056

11 Wang C, Youle R J. The role of mitochondria in apoptosis*. Annu Rev Genet, 2009, 43: 95-118

12 Alton M, Taketo T. Switch from BAX-dependent to BAX-independent germ cell loss during the development of fetal mouse ovaries. J Cell Sci, 2007, 120: 417-424

13 Cook M S, Coveney D, Batchvarov I, et al. BAX-mediated cell death affects early germ cell loss and incidence of testicular teratomas in 
Dnd1 (Ter/Ter) mice. Dev Biol, 2009, 328: 377-383

14 Tsuda M, Sasaoka Y, Kiso M, et al. Conserved role of nanos proteins in germ cell development. Science, 2003, 301: 1239-1241

15 Youngren K K, Coveney D, Peng X, et al. The Ter mutation in the dead end gene causes germ cell loss and testicular germ cell tumours. Nature, 2005, 435: 360-364

16 Hsu S Y, Lai R J, Finegold M, et al. Targeted overexpression of Bcl-2 in ovaries of transgenic mice leads to decreased follicle apoptosis, enhanced folliculogenesis, and increased germ cell tumorigenesis. Endocrinology, 1996, 137: 4837-4843

17 Gordo A C, Rodrigues P, Kurokawa M, et al. Intracellular calcium oscillations signal apoptosis rather than activation in in vitro aged mouse eggs. Biol Reprod, 2002, 66: 1828-1837

18 Perez G I, Robles R, Knudson C M, et al. Prolongation of ovarian lifespan into advanced chronological age by Bax-deficiency. Nat Genet, 1999, 21: 200-203

19 Kujjo L L, Laine T, Pereira R J, et al. Enhancing survival of mouse oocytes following chemotherapy or aging by targeting Bax and Rad51. PLoS ONE, 2010, 5: e9204

20 Broekmans F J, Knauff E A, te Velde E R, et al. Female reproductive ageing: current knowledge and future trends. Trends Endocrinol Metab, 2007, 18: 58-65

21 Faddy M J, Gosden R G. A model conforming the decline in follicle numbers to the age of menopause in women. Hum Reprod, 1996, 11: 1484-1486

22 Reddy P, Zheng W, Liu K. Mechanisms maintaining the dormancy and survival of mammalian primordial follicles. Trends Endocrinol Metab, 2009, 21: 96-103

23 Reddy P, Adhikari D, Zheng W, et al. PDK1 signaling in oocytes controls reproductive aging and lifespan by manipulating the survival of primordial follicles. Hum Mol Genet, 2009, 18: 2813-2824

24 Iwanami A, Cloughesy T F, Mischel P S. Striking the balance between PTEN and PDK1: it all depends on the cell context. Genes Dev, 2009, 23: 1699-1704

25 Reddy P, Liu L, Adhikari D, et al. Oocyte-specific deletion of Pten causes premature activation of the primordial follicle pool. Science, 2008, 319: 611-613

26 White Y A, Woods D C, Takai Y, et al. Oocyte formation by mitotically active germ cells purified from ovaries of reproductive-age women. Nat Med, 2012, 18: 413-421

27 Zou K, Yuan Z, Yang Z, et al. Production of offspring from a germline stem cell line derived from neonatal ovaries. Nat Cell Biol, 2009, 11: 631-636

28 Greenfeld C, Flaws J A. Renewed debate over postnatal oogenesis in the mammalian ovary. Bioessays, 2004, 26: 829-832

29 Liu Y, Wu C, Lyu Q, et al. Germline stem cells and neo-oogenesis in the adult human ovary. Dev Biol, 2007, 306: 112-120

30 Gougeon A. Is neo-oogenesis in the adult ovary, a realistic paradigm? Gynecol Obstet Fertil, 2010, 38: 398-401

31 De Felici M. Germ stem cells in the mammalian adult ovary: considerations by a fan of the primordial germ cells. Mol Hum Reprod, 2010, 16: 632-636

32 Notarianni E. Reinterpretation of evidence advanced for neo-oogenesis in mammals, in terms of a finite oocyte reserve. J Ovarian Res, 2011, 4: 1

33 Zhang H, Zheng W, Shen Y, et al. Experimental evidence showing that no mitotically active female germline progenitors exist in postnatal mouse ovaries. Proc Natl Acad Sci USA advance online publication 9 July 2012; doi: 10.1073/pnas.1206600109

34 Mukhopadhyay A, Tissenbaum H A. Reproduction and longevity: secrets revealed by C. elegans. Trends Cell Biol, 2007, 17: 65-71

35 Blomquist G E. Trade-off between age of first reproduction and survival in a female primate. Biol Lett, 2009, 5: 339-342

36 Attisano A, Moore A J, Moore P J. Reproduction-longevity trade-offs reflect diet, not adaptation. J Evol Biol, 2012, 25: 873-880

37 Westendorp R G, Kirkwood T B. Human longevity at the cost of reproductive success. Nature, 1998, 396: 743-746

38 Muller H G, Chiou J M, Carey J R, et al. Fertility and life span: late children enhance female longevity. J Gerontol A Biol Sci Med Sci,
2002, 57: B202-B206

39 Helle S, Lummaa V, Jokela J. Are reproductive and somatic senescence coupled in humans? Late, but not early, reproduction correlated with longevity in historical Sami women. Proc Biol Sci, 2005, 272: 29-37

40 Lahdenpera M, Lummaa V, Helle S, et al. Fitness benefits of prolonged post-reproductive lifespan in women. Nature, 2004, 428: 178-181

41 Hsin H, Kenyon C. Signals from the reproductive system regulate the lifespan of C. elegans. Nature, 1999, 399: 362-366

42 Mitteldorf J. Female fertility and longevity. Age (Dordr), 2010, 32: 79-84

43 Lycett J E, Dunbar R I, Voland E. Longevity and the costs of reproduction in a historical human population. Proc Biol Sci, 2000, 267: 31-35

44 Kachel A F, Premo L S, Hublin J J. Grandmothering and natural selection. Proc Biol Sci, 2010, 278: 384-391

45 Berman J R, Kenyon C. Germ-cell loss extends C. elegans life span through regulation of DAF-16 by kri-1 and lipophilic-hormone signaling. Cell, 2006, 124: 1055-1068

46 Arantes-Oliveira N, Apfeld J, Dillin A, et al. Regulation of life-span by germ-line stem cells in Caenorhabditis elegans. Science, 2002, 295: 502-505

47 Kenyon C, Chang J, Gensch E, et al. A C. elegans mutant that lives twice as long as wild type. Nature, 1993, 366: 461-464

48 Kenyon C. The first long-lived mutants: discovery of the insulin/IGF-1 pathway for ageing. Philos Trans R Soc Lond B Biol Sci, 2010, 366: 9-16

49 Luo S, Kleemann G A, Ashraf J M, et al. TGF-beta and insulin signaling regulate reproductive aging via oocyte and germline quality maintenance. Cell, 2010, 143: 299-312

50 Luo S, Shaw W M, Ashraf J, et al. TGF-beta Sma/Mab signaling mutations uncouple reproductive aging from somatic aging. PLoS Genet, 2009, 5: e1000789

51 Huang C, Xiong C, Kornfeld K. Measurements of age-related changes of physiological processes that predict lifespan of Caenorhabditis elegans. Proc Natl Acad Sci USA, 2004, 101: 8084-8089

52 Hughes S E, Evason K, Xiong C, et al. Genetic and pharmacological factors that influence reproductive aging in nematodes. PLoS Genet, 2007, 3: e25

53 Flatt T, Min K J, D'Alterio C, et al. Drosophila germ-line modulation of insulin signaling and lifespan. Proc Natl Acad Sci USA, 2008, 105: 6368-6373

54 Ghazi A, Henis-Korenblit S, Kenyon C. A transcription elongation factor that links signals from the reproductive system to lifespan extension in Caenorhabditis elegans. PLoS Genet, 2009, 5: e1000639

55 Hamatani T, Falco G, Carter M G, et al. Age-associated alteration of gene expression patterns in mouse oocytes. Hum Mol Genet, 2004, 13: 2263-2278

56 Steuerwald N M, Bermudez M G, Wells D, et al. Maternal age-related differential global expression profiles observed in human oocytes. Reprod Biomed Online, 2007, 14: 700-708

57 Li Q, Agno J E, Edson M A, et al. Transforming growth factor beta receptor type 1 is essential for female reproductive tract integrity and function. PLoS Genet, 2011, 7: e1002320

58 Middlebrook B S, Eldin K, Li X, et al. Smad1-Smad5 ovarian conditional knockout mice develop a disease profile similar to the juvenile form of human granulosa cell tumors. Endocrinology, 2009, 150: 5208-5217

59 McCormick M A, Tsai S Y, Kennedy B K. TOR and ageing: a complex pathway for a complex process. Philos Trans R Soc Lond B Biol Sci, 2010, 366: 17-27

60 Russell R C, Fang C, Guan K L. An emerging role for TOR signaling in mammalian tissue and stem cell physiology. Development, 2011, 138: 3343-3356

61 Laplante M, Sabatini D M. mTOR signaling at a glance. J Cell Sci, 2009, 122: 3589-3594

62 Martelli A M, Chiarini F, Evangelisti C, et al. Two hits are better than one: targeting both phosphatidylinositol 3-kinase and mamma- 
lian target of rapamycin as a therapeutic strategy for acute leukemia treatment. Oncotarget, 2012, 3: 371-394

63 Cloughesy T F, Yoshimoto K, Nghiemphu P, et al. Antitumor activity of rapamycin in a Phase I trial for patients with recurrent PTENdeficient glioblastoma. PLoS Med, 2008, 5: e8

64 Guertin D A, Sabatini D M. Defining the role of mTOR in cancer. Cancer Cell, 2007, 12: 9-22

65 Laplante M, Sabatini D M. mTOR signaling in growth control and disease. Cell, 2012, 149: 274-293

66 Wolf G. Calorie restriction increases life span: a molecular mecha- nism. Nutr Rev, 2006, 64: 89-92

67 Everitt A V, Le Couteur D G. Life extension by calorie restriction in humans. Ann N Y Acad Sci, 2007, 1114: 428-433

68 Harrison D E, Strong R, Sharp Z D, et al. Rapamycin fed late in life extends lifespan in genetically heterogeneous mice. Nature, 2009, 460: 392-395

69 Selesniemi K, Lee H J, Muhlhauser A, et al. Prevention of maternal aging-associated oocyte aneuploidy and meiotic spindle defects in mice by dietary and genetic strategies. Proc Natl Acad Sci USA, 2011, 108: 12319-12324

Open Access This article is distributed under the terms of the Creative Commons Attribution License which permits any use, distribution, and reproduction in any medium, provided the original author(s) and source are credited. 